INVESTIGACIONES

\title{
Interculturalidad en la formación docente: Un aporte desde las voces de personas de los pueblos originarios*
}

\author{
Interculturality in teacher education: \\ A contribution from indigenous peoples' voices
}

\author{
Nolfa Ibáñez Salgado, na María Figueroa Espínola ${ }^{b}$ \\ M. Soledad Rodríguez $z^{c}$ Alvaro Aros Nuñez \\ ${ }^{a}$ Universidad Metropolitana de Ciencias de la Educación. \\ Correo electrónico: nolfa.ibanez@umce.cl \\ ${ }^{\mathrm{b}}$ Universidad Metropolitana de Ciencias de la Educación. \\ Correo electrónico: ana.figueroa@umce.cl. \\ ${ }^{c}$ Universidad Metropolitana de Ciencias de la Educación. \\ Correo electrónico: m_soledad.rodriguez@umce.cl. \\ ${ }^{\mathrm{d}}$ Universidad Metropolitana de Ciencias de la Educación. \\ Correo electrónico: alvaroaros.1993@gmail.com.
}

\begin{abstract}
RESUMEN
El artículo informa de una investigación que analiza discursos de personas mapuche y aymara, en relación a la formación docente. El objetivo es contribuir a la formación para la atención pedagógica a la diversidad cultural en las carreras de Pedagogía, cuestión que consideramos urgente. Se presenta una visión general del contexto actual, los principales fundamentos conceptuales y epistemológicos en los que se basa el estudio y sus lineamientos metodológicos. Los resultados preliminares muestran una casi total correspondencia entre las conceptualizaciones, valoraciones y expectativas sobre la formación docente de los distintos actores: profesores, educadores tradicionales, estudiantes, apoderados y familiares, tanto mapuche como aymaras.
\end{abstract}

Palabras clave: diversidad cultural, Educación superior, percepción de los actores.

\begin{abstract}
This article reports on a study that analyzed the discourses of Mapuche and Aymara people in relation to teacher education. Our aim is to contribute to the inclusion of specific training in pedagogical attention to cultural diversity in formal processes higher education for teachers, an issue that we consider urgent. This article presents a general view of the current context, the main conceptual and epistemological foundations on which the study is based, and its methodological guidelines. The results of the first levels of analysis show a nearly complete correspondence between the conceptualizations, evaluations, and expectations about teacher education held by the different actors consulted: teachers, traditional educators, students, parents, and relatives, both Mapuche and Aymara.

Keywords: cultural diversity, higher education, actors' perceptions.
\end{abstract}


Estudios Pedagógicos XLIV, $\mathrm{N}^{\circ}$ 1: 225-239, 2018

INTERCULTURALIDAD EN LA FORMACIÓN DOCENTE: UN APORTE DESDE LAS VOCES DE PERSONAS DE LOS PUEBLOS ORIGINARIOS

\section{INTRODUCCIÓN}

En el marco de una línea de investigación sobre Diversidad, desde hace más de una década hemos indagado sobre y en los modos de conocer y aprender de personas de culturas distintas a la dominante o mayor, específicamente mapuche y aymara, con el propósito de contribuir a la comprensión y valoración de la diversidad cultural en el ámbito educativo en general y en la formación docente en particular. El desarrollo de esta línea nos ha permitido constatar, como observadores pertenecientes a la cultura mayor, que el modo de hacer las cosas de las personas indígenas con las que hemos compartido, conlleva lógicas distintas y en algunos casos opuestas a las de la escuela chilena y, en general, a las que imperan en la sociedad mayor y orientan los programas de desarrollo de la política pública que se focalizan en la población indígena. Del mismo modo, mediante esta prolongada relación con personas de los pueblos originarios, hemos conocido y comprendido sus concepciones sobre la escuela y la educación, en su sentido amplio.

El presente artículo es parte de una investigación, cuyo objetivo es contribuir a la formación para la atención pedagógica a la diversidad cultural en las carreras de Pedagogía, a partir de develar las concepciones, valoraciones y expectativas que tienen personas pertenecientes a nuestros pueblos originarios sobre la formación docente. Asumimos el concepto de interculturalidad como diálogo entre culturas (Etxeberría 2003), que debe darse en igualdad de condiciones para hacer posible que las personas que pertenecen a culturas minoritarias se sientan reconocidas en su diferencia cultural.

Se analizan las conceptualizaciones, valoraciones y expectativas sobre la formación docente, presentes en los discursos de personas mapuche y aymaras, colaboradores en cuatro investigaciones educacionales desarrolladas entre 2006 y 2014 ${ }^{1}$. Estos discursos corresponden a entrevistas individuales y grupales, grupos focales de conversación y Talleres de coconstrucción. Dichas investigaciones, si bien se focalizaron en la implementación curricular en educación parvularia y básica, en las concepciones de los participantes sobre aprendizaje, educación y escuela y en la pertinencia que para ellos tiene el programa de Educación Intercultural Bilingüe, muchos de los discursos recogidos aluden explícita o implícitamente a la formación docente, ámbito en el cual la mayoría de los participantes deposita sus expectativas de cambio en relación a la aceptación de la diversidad cultural por parte de la sociedad chilena.

La pertinencia de este rescate de las voces de personas mapuche y aymaras, responde a la necesidad de generar nuevo conocimiento que aporte a los cambios estructurales que se están proponiendo para el sistema educativo chileno, en un ámbito considerado de crucial importancia: la atención pedagógica a la diversidad del estudiantado; en nuestro caso, a la diversidad cultural de niñas, niños y jóvenes. Si bien la atención educativa a la diversidad es reiteradamente relevada en el discurso educativo general sobre el mejoramiento de la calidad de la educación y en el particular de las instituciones formadoras, la investigación muestra que las competencias profesionales que la abordan serían las que tienen menor presencia en el aula escolar (Alegría y Cisternas 2003; Flórez 1994; Ibáñez 2014; Jáuregui Carrasco y Montes, 2003; Valenzuela 2006).

Aquí presentamos una visión general del contexto del estudio, sus principales fundamentos conceptuales y epistemológicos, los lineamientos metodológicos y algunos resultados preliminares.

Proyectos Fondecyt N ${ }^{\circ} 1060230 / 2006-2009$ y N¹111030/2011-2014. Proyectos Fonide N55/2008-2009 y F811349/2014-2015. 


\section{CONTEXTUALIZACIÓN}

Actualmente, en América Latina existen 826 pueblos indígenas que se caracterizan por una gran heterogeneidad; diversidad demográfica, social, territorial y política. En este último decenio, ha habido un incremento en la población indígena en la región, la cual corresponde a una población total aproximada de 45 millones que, en términos porcentuales, sería el 8\% de la población total (Comisión Económica para América Latina y El Caribe, CEPAL 2014). Pese a esta realidad, la homogeneidad cultural instalada desde la colonización y propiciada por los Estados principalmente a través de los sistemas educativos, ha impedido la visibilización y reconocimiento de la gran diversidad cultural que caracteriza a la Región. Las reivindicaciones políticas y culturales de los pueblos originarios han cuestionado los modelos educativos imperantes, que se han implementado a través de estructuras curriculares construidas desde una perspectiva epistemológica técnica-instrumental propia de occidente (Turra 2012), que se centra en la enseñanza de saberes parcelados y se sustenta en modelos ajenos a la visión de mundo de los pueblos originarios. (Díaz y Druker 2007; Ibáñez, Díaz, Druker y Rodríguez 2012; Pinto 2008; Quilaqueo 2007). Chile se ubica dentro de los 5 países de la región con mayor presencia de personas que pertenecen a pueblos indígenas, 1.805.243 personas, que corresponden al 11\% de la población total. De acuerdo a los resultados del Censo 2012, publicados y retirados por problemas de calidad (CEPAL 2016), la mayor población indígena correspondería a los pueblos mapuche y aymara, con un $84,11 \%$ y un $6,25 \%$ respectivamente. En las regiones de la Araucanía y los Ríos vive el 53,54\% de la población mapuche, en tanto el 74,6\% de la población aymara reside en las regiones de Arica-Parinacota y Tarapacá las dos regiones del extremo norte del país que limitan con Perú y Bolivia.

Las escuelas situadas en lugares con mayor densidad poblacional indígena son las que tienen menores resultados en las pruebas estandarizadas nacionales (Ministerio de Educación, Mineduc 2014) y mayores índices de pobreza (Encuesta de Caracterización Socioeconómica, CASEN 2015). Un estudio reciente constata que los estudiantes de escuelas de alta composición indígena así como las escuelas de comunas donde existe una mayor segregación indígena escolar, presentan una asociación negativa con los resultados de las pruebas nacionales SIMCE (Webb, Canales y Becerra 2017). En lo que concierne a educación superior, el Estado no destina fondos directos o concursables a las universidades o institutos profesionales públicos para la creación de programas o carreras interculturales o con un enfoque intercultural, unido a que la mayoría de estas instituciones no ha considerado el desarrollo de programas con este enfoque y solo dos universidades en Chile tienen carreras de pedagogía declaradas interculturales. Como señala el estudio de Mora, las pocas instituciones de educación superior que mantienen programas interculturales, ya sea en forma de apoyo a estudiantes o de redes de académicos e investigadores en el área de interculturalidad, no tendrían como respaldo una política institucional al respecto ni presupuesto regular destinado a ello (Mora (2011). Uno de los puntos conflictivos que señala esta autora,

es el de los recursos a invertir para el establecimiento de iniciativas de este tipo. En general, se hace en base a proyectos de colaboración con la CONADI [Corporación de Desarrollo Indígena] u organismos internacionales (Fundación Ford, Kellog's, UNESCO y otras), pensados como transitorios, de pocos recursos y sin un compromiso 
económico mayor por parte de la misma institución, lo que los hace altamente vulnerables y de alta caducidad. (Mora 2011).

La actual Reforma Educacional tiene como sus principales objetivos la reconstrucción de la educación pública y la mejora en la calidad de la misma. La educación pública en Chile fue severa y multidimensionalmente precarizada por la dictadura militar, que traspasó los establecimientos educacionales a las municipalidades dirigidas por alcaldes designados por ese mismo gobierno dictatorial, disminuyendo en forma considerable el presupuesto para educación, al mismo tiempo que promovió la creación de escuelas por parte de privados, las que podían hasta hace muy poco recibir dinero del Estado y tener fines de lucro (Claude 2011; Mizala 2007; Oficina Regional de la Internacional de la Educación para América Latina, ORIEAL 2003; Paredes y Pinto 2009). Recién hoy, después de más 27 años del término del gobierno dictatorial y gracias a las reformas impulsadas por los sucesivos gobiernos democráticos, especialmente los de la presidenta Bachelet con su enfoque de derechos, podemos decir que se han logrado sentar las bases para que los objetivos de la Reforma puedan ser alcanzados en el mediano plazo. En efecto, hoy la educación pública chilena tiene un presupuesto preferencial, vuelve a ser gratuita para los estudiantes de enseñanza básica y media, el lucro ya no es permitido, existe una Ley de Inclusión que prohíbe la selección en los establecimientos educacionales que reciben aportes del Estado y se ha aprobado recientemente la Ley de desmunicipalización de las escuelas y liceos públicos, los que pasarán a depender de agencias locales que responderán nuevamente al Ministerio de Educación, entre otros avances. Se enfatiza en esta Reforma la valoración de las diferencias, la igualdad de derechos, el fortalecimiento de las comunidades educativas, el liderazgo de los actores y el mejoramiento continuo. También se reconoce el hecho que la sociedad chilena es pluricultural y que el currículo nacional "debe partir de la base que los pueblos indígenas existentes en el país son portadores de culturas específicas y, en consecuencia, es necesario reconocer que la diversidad y pluriculturalidad constituyen la identidad nacional" (Mineduc 2016).

Para lograr el propósito de reposicionar una educación pública de calidad, el país requiere contar con docentes que, más allá de los necesarios conocimientos disciplinares y didácticos, estén también formados en un saber pedagógico que comprenda y valore la diversidad, con competencias para generar espacios de democratización en la institución escolar y para propiciar una disposición emocional favorable para el aprendizaje en la implementación curricular en sus respectivas salas de clase, considerando las características particulares de sus contextos de desempeño profesional y de su grupo de estudiantes, lo que conlleva la necesaria comprensión de que los niños, niñas y jóvenes, con independencia de su edad, origen social, económico o étnico, traen un mundo a la mano que debe ser considerado y valorado como base de sus nuevos aprendizaje. Estas serían competencias profesionales que debiese construir todo docente en su formación teórica y práctica, entendidas como la operacionalización de saberes en un contexto situacional determinado. La importancia de estos aspectos ha sido relevada desde hace varios años por distintos autores y también por recomendaciones nacionales e internacionales que reconocen que la atención pedagógica a la diversidad de los estudiantes, la valoración de sus experiencias previas y el propiciar un estilo de interacción grato en el aula, no sólo mejoraría la calidad de sus aprendizajes, sino también su desarrollo como personas (Cassasus 2007; Comisión Europea 2002; Ibáñez 2002; Ibáñez, Barrientos, Delgado, Figueroa y Geisse 
2011; Maturana 1990; Mineduc 2005; Organización para la Cooperación y el Desarrollo Económico, OCDE 2010; OCDE 2004; Unesco 2000, 2008).

\section{ASPECTOS CONCEPTUALES Y EPISTEMOLÓGICOS}

La investigación que desarrollamos tiene el propósito de contribuir a que la sociedad mayor comprenda lo que piensan y sienten personas de culturas minoritarias respecto a la formación de profesores en Chile y a la inclusión de la interculturalidad en dicha formación, para aportar a la inclusión educacional y social de las personas de los pueblos originarios y al trabajo pedagógico con la diversidad cultural en general. Para este propósito, consideramos imprescindible que la formación docente se haga cargo de la responsabilidad que al respecto le cabe, por cuanto sus currículos, en general, no han considerado la interculturalidad (Leiva 2009, Quintriqueo et al. 2014) y como señala Quilaqueo, "han obviado los saberes y conocimientos indígenas" (Quilaqueo 2007). En efecto, la academia no considera modos distintos de construcción y de validación de conocimientos, aun cuando aquello significaría un aporte valioso a la formación de profesores; en expresión de Nanculef.

Nosotros no aprendemos "cómo hacer las cosas", sino que simplemente aprendemos a "hacer las cosas", es lo que nuestros hermanos aymara denominan Qhapag-Ñan. Este es un salto epistemológico que la sociedad chilena y los pedagogos de la educación formal en Chile debieran considerar como un gran aporte de la sociedad indígena a la sociedad en general, sobre todo en este tiempo" (Ñanculef 2016).

A diferencia de lo que ocurre en las sociedades occidentales, las personas indígenas no aprenden primero sobre la vida para después vivirla. En las sociedades indígenas el conocimiento tiene que ver con su relevancia y la ampliación de ese conocimiento se desarrolla a través de la experiencia, como parte del crecimiento: "Knowledge is acquired because en when it is relevant, and increases through experience as part of growing to maturity" (Crickmay 2002).

Para la formación de profesores, construir un currículo que contemple la diversidad cultural, implica competencias diseñadas para acoger pedagógicamente los distintos sentidos y significados que traen las y los estudiantes que provienen de culturas distintas a la dominante o mayor; también obliga a enfatizar lo experiencial y la relación dialógica entre la teoría y la práctica, para cautelar una inclusión efectiva, que no quede en declaraciones alejadas de su implementación en el aula. Esto constituye un gran desafío, por cuanto sabemos que la teoría que se aprende y acepta en la formación y la práctica pedagógica que se desarrolla en los establecimientos educacionales constituyen "mundos amplios e intrínsecamente complejos internamente" (Alvarez 2012). Estos mundos estarían separados por

Un abismo en el fondo del cual acaban no pocos docentes incapaces de superar la distancia entre los alejados bordes de la teoría y de la práctica [...] Y hasta puede ser considerada como una fractura epistemológica inevitable entre la búsqueda y la difusión del conocimiento en la Academia y su utilización escolar para formar a niños y jóvenes (Romero, García y Rozada 2007). 
Además de esta dificultad no menor, sabemos también que para implementar modelos de cambio paradigmático se requiere que todos los ámbitos de la educación se involucren. Hoy se reconoce que la función docente va mucho más allá de la transmisión de contenidos, se otorga relevancia al saber pedagógico del profesor como entidad epistemológica que le es propia, se reconoce su saber experiencial y su capacidad transformadora y formadora, se comprende que su saber es situado y que debe analizarse considerando el resto de las condiciones de su contexto de desempeño profesional, tanto académicas como administrativas y políticas (Moon 2002; Ibáñez 2014; Tardif y Lessard 2000; Tardif 2004), por lo que el rol que cumplen las y los docentes de las instituciones formadoras de profesores es fundamental para intencionar la transformación de lógicas instaladas en el sistema escolar, lo que también conlleva hacerse cargo de la responsabilidad histórica que le cabe a la formación docente en la reproducción de las lógicas oficiales y de los saberes hegemónicos de la sociedad dominante en la educación de los niños, niñas y jóvenes que pertenecen a culturas minoritarias.

Dado el nuevo escenario educacional en Chile, que incluye la acreditación obligatoria de las carreras de pedagogía, las instituciones que forman profesores están abocadas a la modernización de sus procesos y a la formulación de innovaciones que consideren los nuevos escenarios socioeducativos para dar cumplimiento a la misión que les compete en la sociedad, lo que configura un escenario propicio para los cambios en un momento histórico de reformas en educación. La formación docente entonces, para hacer coherente el discurso que adopta y difunde con la práctica concreta que realiza, tiene el importante desafío de diseñar competencias que permitan al futuro profesor generar las mismas oportunidades para el aprendizaje de todas y todos, indígenas y no indígenas, chilenos y extranjeros. Creemos que uno de los principales obstáculos para este abordaje lo constituye la imagen colectiva que tendría la sociedad chilena sobre las personas indígenas y, en general, sobre el otro diferente. La investigación en Chile revela que la discriminación hacia la diferencia constituye una realidad (Observatorio Ciudadano 2013; Programa de las Naciones Unidas para el Desarrollo 2013; Instituto Nacional de Derechos Humanos 2011; Redón, Toledo y Rubio 2009; Naciones Unidas 2009, 2013; Alarcón 2005; Aymerich, Canales y Vivanco 2003; Magendzo 2000), mostrando que la aceptación de la diversidad está lejos de caracterizar a la escuela y al conjunto de nuestra sociedad. En lo concerniente a los pueblos originarios, los prejuicios tendrían que ver

con el racismo de la desigualdad que en el caso de Chile se asocian al llamado prejuicio civilizatorio (...). Este prejuicio sitúa la cultura de las etnias como obstáculo al desarrollo y residuo histórico destinado a desaparecer en los procesos de modernización social y asimilación cultural (Aymerich, Canales y Vivanco 2003).

Esto hace aún más compleja la tarea formativa, pero al mismo tiempo, más relevante para el país, por cuanto la profesión docente es la que tendría mejores posibilidades de incidir en el cambio de esa imagen colectiva.

La incorporación de la interculturalidad en la formación docente implica aceptar como legítimos modos distintos de construcción y validación de conocimientos, ampliando el saber pedagógico de quienes serán profesores y profesoras, y preparándolos para acoger y valorar la diversidad cultural con la que se encontrarán en su desempeño profesional. Esta perspectiva importa también para el estudiantado un potencial de aprendizaje sobre la 
aceptación del otro en la convivencia (Maturana 1992), lo que podría contribuir a modificar positivamente, en un futuro próximo, la imagen que tiene la sociedad chilena sobre el otro diferente. En palabras de Habermas,

los participantes en la interacción, al entenderse entre sí sobre una situación, se mueven en una tradición cultural, de la que hacen uso a la vez que la renuevan (...) se apoyan en pertenencias a grupos sociales y refuerzan simultáneamente la integración de éstos; y el niño, al participar en interacciones con personas de referencia que actúan competentemente, internaliza las orientaciones valorativas de su grupo social y adquiere capacidades generalizadas de acción (Habermas 1994).

Este enfoque intercultural debe considerar también, como un aspecto importante, el aporte de los estudiantes a las decisiones y reflexiones del docente. En un estudio realizado por Mercado sobre saberes docentes, la autora hace notar que en la perspectiva de investigación desarrollada a partir de los 80 en Inglaterra y Estados Unidos -por autores como Yinger, Schulman y Caldrehead-, que ha indagado en el conocimiento que tienen los docentes sobre la enseñanza que imparten, "no resulta relevante el papel de los alumnos en los procesos de decisión docente" (Mercado 2002), lo que es contradictorio con sus propios hallazgos, los cuales relevan la importancia de los estudiantes en el proceso de enseñanza:

a través de todo el estudio se muestra la forma como los alumnos están siempre presentes en el diálogo que los maestros establecen con diferentes voces durante la enseñanza [...] en todas las decisiones que los maestros toman antes y durante la enseñanza y en muchas de sus más importantes dudas y reflexiones" (Mercado 2002).

Los estudiantes de cada grupo, según sus características y el contexto relacional en el cual tiene lugar la situación de aprendizaje-enseñanza, inciden en las decisiones que toma el profesor. Si bien "frente a la diversidad de sus alumnos, el docente debe tener una comprensión flexible y polifacética, que le permita poder impartir explicaciones alternativas a los mismos conceptos o principios" (Shulman 1985), esas explicaciones alternativas sólo pueden tener sentido para el o la estudiante si la comprensión flexible y polifacética implica acoger los significados previos que él o ella trae sobre el contenido de la clase y que podrían ser diferentes en todo o en parte a los del profesor, si el estudiante proviene de otra cultura. Hacerse cargo de la legitimidad de esos conocimientos, valorarlos e incorporarlos a las temáticas de la clase, resulta también un aprendizaje para quien enseña y necesariamente cambia el estilo relacional habitual de las clases directivas centradas en el docente, estilo que las y los profesores chilenos parecen seguir conservando, como muestra el estudio realizado por Preiss et al. sobre los videos de evaluación docente en el segundo ciclo básico (Preiss et al. 2014). Si entendemos el aprendizaje como un fenómeno de transformación en la convivencia (Maturana 1992), la aceptación de la diversidad de construcciones de mundo -significados y sentidos- que traen los estudiantes al aula, permitiría que el grupo conozca, respete y valore, saberes y modos de hacer las cosas que son distintos a aquellos aprendidos en la propia cultura e implicaría trasladar, aunque sea en parte, el foco de la clase desde el profesor a los estudiantes, modificando la estructura interaccional habitual 
del aula y, por tanto, cambiando el fluir relacional de esa convivencia y las acciones de los participantes en la interacción. Como la construcción de conocimiento depende del modo en el que las personas categorizan y otorgan significado a sus experiencias (Long 1992), la atención pedagógica a la diversidad cultural del estudiantado entendida de este modo, generaría nuevo conocimiento para docentes y estudiantes, por cuanto "el conocimiento siempre se adquiere en la convivencia (...) el conocimiento siempre tiene que ver con las acciones" (Maturana 1990).

Nuestro trabajo pretende visibilizar las conceptualizaciones, valoraciones y expectativas sobre la formación docente que tienen personas de culturas distintas a la dominante o mayor, para contribuir a la innovación que haga posible la incorporación efectiva de la interculturalidad en la formación de profesores, y por tanto, a situar la atención a la diversidad, y a la diversidad cultural en particular, como uno de los aspectos centrales en la construcción del saber pedagógico del docente, asumiendo que un proyecto intercultural para la educación superior debe considerar, en primer lugar, que su pertinencia sea validada por quienes pertenecen a otras culturas y "no buscar la asimilación ni redundar en la fragmentación de las culturas e identidades de los estudiantes, sino que por el contrario, fortalecer estas identidades culturales, mediante la validación del proceso de construcción desde lo propio" (Druker 2010).

\section{ASPECTOS METODOLÓGICOS}

La metodología del estudio se enmarca en un enfoque general cualitativo), considerando la particularidad del espacio y tiempo donde se producen (Goetz y Le Compte 1998).

El diseño metodológico consideró en su primera etapa la definición del corpus a analizar: discursos que aluden explícita o implícitamente a la formación de profesores. Estos discursos fueron recogidos en entrevistas, grupos de conversación y talleres de coconstrucción, entre 2006 y 2014, en el marco de proyectos de investigación, con estricto apego al protocolo ético correspondiente, grabados en audio y transcritos para su análisis. La primera etapa del diseño consistió en la identificación del material que constituiría el corpus definitivo, según se distinguiera en las expresiones vertidas por los participantes una relación explícita o implícita con la formación docente. Este corpus definitivo lo conformaron los discursos de 48 personas indígenas, 40 mapuche y 8 aymaras; de los cuales 16 son docentes, 13 educadores tradicionales, 9 apoderados, 5 estudiantes y 5 familiares de distintas generaciones. En una segunda etapa, el material seleccionado se separó por actor o rol (estudiante, docente, educador/a tradicional, apoderado/a o familiar de un/a estudiante), identificando su pertenencia a la cultura mapuche o aymara. Para el primer nivel de análisis del contenido de estos discursos se establecieron tres dimensiones: Conceptualizaciones, Valoraciones y Expectativas. De acuerdo a los resultados del primer nivel de análisis, para un segundo nivel se definieron tres categorías al interior de cada dimensión: a) Sobre implementación curricular; b) Sobre modos de relación interpersonal y c) Sobre contenidos curriculares. Los contenidos de cada una de estas categorías se separaron en explícitos e implícitos, por actor o rol, para efectuar, posteriormente, un análisis de correspondencia entre ellos, que constituyó el tercer nivel de análisis. En todo el proceso se utilizaron procedimientos de codificación, condensación e interpretación de significados (Kvale 2011; Gibbs 2012) acompañadas de dispositivos de triangulación entre 
investigadores del equipo con el fin de cautelar la fiabilidad y validez de los hallazgos. Complementariamente, se realizaron sesiones de trabajo con dos colaboradores mapuche hablantes de su lengua originaria y que participaron en los estudios previos ${ }^{2}$, en las cuales se discutieron los resultados de cada nivel de análisis, con el propósito de validar la interpretación hecha por el equipo para asegurar la pertinencia de los resultados. Este artículo informa los principales resultados correspondientes al primer nivel de análisis.

\section{ALGUNOS RESULTADOS}

Se señalan resultados del primer nivel de análisis, correspondientes a las tres dimensiones consideradas: Conceptualizaciones, Valoraciones y Expectativas.

En relación a las conceptualizaciones sobre la formación docente y lo que ésta debiese implicar, las mayormente compartidas refieren a la necesidad de que la sociedad chilena comprenda la interculturalidad como un valor para todos, independientemente de la cultura a la que se pertenezca, porque el aprendizaje intercultural es bidireccional. Mirado desde la perspectiva de la interacción comunicativa, este aprendizaje bidireccional correspondería a lo que permite reforzar pero también renovar las tradiciones culturales (Habermas 1994). La interculturalidad en general, sería más necesaria para las personas de la cultura dominante o mayor, puesto que tanto las personas mapuche y aymara se consideran a sí mismas interculturales. No obstante lo anterior, otorgan gran importancia a la inclusión de la interculturalidad en la formación docente y critican el hecho que la formación docente invisibilice otras culturas y no prepare para recibir a estudiantes que tienen distintos aprendizajes previos y distintos contextos, cuestión que ha sido ya señalada por diversos autores, como por ejemplo Quilaqueo y Ñanculef, entre otros (Quilaqueo 2007; Nanculef 2016). Las siguientes expresiones ejemplifican lo anterior:

- Si es winka [profesor no indígena] tiene que saber lo básico porque también él puede aprender con los niños (apoderada mapuche).

-El niño no mapuche debe conocer la cultura mapuche, para una sociedad más justa (educadora tradicional).

- El tema de educación intercultural no nos puede ser destinado a nosotros porque nosotros somos interculturales [...] yo creo que nosotros como mapuche tenemos que abocarnos a una educación destinada a fortalecer nuestra lengua, nuestra cultura, etcétera, y a las personas que son chilenas, ... la educación intercultural (apoderado).

- El profesor tiene que saber. Por ejemplo, el niño que vive en el altiplano tiene enseñanzas distintas a la que tiene acá. Tiene responsabilidades diferentes [,,,] el niño que está arriba tiene que, antes de irse a la escuela o en la semana, ir a pastorear o tiene que ir a buscar el agua o buscar leña, hacer todas esas cosas. El niño que vive en la ciudad con suerte lava su plato (apoderada aymara). 
- La sociedad chilena debe comprender que la cultura mapuche es presente, no pasado [...] si los profesores aprendieran en la universidad cultura mapuche, yo me sentiría reconocido por el Estado chileno (educador tradicional mapuche).

Las valoraciones mayormente compartidas respecto a la formación docente son críticas y aluden principalmente a la falta de preparación y de compromiso de los profesores con lo intercultural. La práctica y la experiencia son para ellos lo más importante para aprender, mientras que los profesores se formarían principalmente en lo teórico, lo que da cuenta de que se tiene claridad respecto a que ellos, en relación a la cultura dominante o mayor, tienen modos distintos de construcción de conocimiento, (Crickmay 2002). Los principios y lógicas presentes en la formación de profesores tendrían como consecuencia que las prácticas docentes habituales generen confusión en sus niños, por cuanto van en sentido opuesto a lo aprendido al interior de la familia y la comunidad; como expresó un profesor "cuando el mapuche enseña en la casa no enseña poniendo el objetivo primero, lo primero que pone el mapuche es el ejemplo“. Existe un sentimiento compartido respecto a que los encargados de elaborar los currículos de formación docente no considerarían las lógicas de enseñanza de otras culturas, lo que se evidencia no sólo en la ausencia de contenidos, sino en el hecho que en esos currículos lo pedagógico está fuertemente estructurado y más ligado a lo teórico que a lo práctico, alejándose de lo experiencial que se considera prioritario en sus culturas. En este caso, la lejanía entre la teoría y la práctica docente contextualizada (Romero, García y Rozada 2007), sería mucho mayor. Como señalaron algunos participantes:

- La cultura mapuche se enseña en procesos lentos, y más autónomos también... en cambio [...] estos programas [de formación docente] lo hacen muy estructurado ... y es para estar encima de él [del estudiante] para que aprenda, y nosotros no lo hacemos así (profesora mapuche).

- Tratan de dividir los temas y en la cultura mapuche es muy complicado dividir los temas porque tú hablas de tierra, de números, de naturaleza, de lenguaje, conocimiento de la luna, o sea, de todo (educador tradicional mapuche).

- La forma de enseñar de algunos profesores es demasiada teórica y no práctica (estudiante aymara).

- Lógicamente el mundo mapuche es espiritual y es racional. Yo no puedo pasar el contenido de un conocimiento que solo sea racional. Tiene que llevar una connotación espiritual (educador tradicional mapuche).

Existe consenso en que la formación docente parece no considerar los aspectos contextuales, en el entendido que el contextualizar no refiere solamente a los contenidos sino también a la forma de trabajo en el aula, según las características de los estudiantes:

a un profesor deberían decirle [enseñarle] construye un material que sea apropiado para ti porque te traen unos libros que están súper descontextualizados de lo que tú debieras trabajar [...] porque todo depende del contexto donde uno esté, primero que nada (profesora mapuche). 
Si bien la formación de profesores entrega contenidos, no daría prioridad a la formación de la persona y como consecuencia, en la escuela se fomentaría la competencia y el individualismo, lo que se opone a las lógicas de colaboración que guían el quehacer de las personas mapuche y aymaras, como ha sido señalado por diversos autores (Ibáñez et al. 2012; Carihuentro 2007, entre otros). Como ejemplo de lo anterior, las siguientes expresiones:

- Cada uno responde su prueba, cada uno hace su trabajo, cada uno hace lo suyo y en el caso de los mapuche siempre ha sido en forma colectiva [...] y uno aprendió en forma colectiva (profesora mapuche)

- Pero hoy día los contenidos son más exigentes y toda la tecnología que uno tiene al alcance les permite... ayuda a los niños, pero en la calidad de persona, el colegio no forja (educador tradicional mapuche)

En relación a las expectativas, se comparte que las culturas mapuche y aymara debiesen tener presencia en la formación de profesores, para posibilitar la aceptación de las culturas minoritarias por parte de la sociedad mayor. En expresión de algunos participantes:

- Lo que falta es más profesionales, profesionales que... que tengan el conocimiento de la lengua y la cultura, o sea, un Educador Tradicional no es hoy en día no es suficiente, o sea, tiene que ver capacitarse, formarse más profesores o aquellas personas que tengan conocimiento, no es lo mismo saber hablar aymara que tener la pedagogía (profesor aymara).

- Y creo que lo otro principal debiera ser que el que se esté educando para profesor..., que en esos 5 años tenga un subsector donde le enseñen o donde se trabaje el ámbito de la cultura mapuche" (profesor mapuche).

- En las universidades, debiera incluirse un cambio de metodología, no sé, en el currículo, que se incorpore conocimiento mapuche para que los estudiantes y los futuros profesores también conozcan quiénes somos (educador tradicional mapuche).

Los resultados preliminares muestran una casi total correspondencia entre lo expresado por los distintos actores en cuanto a las conceptualizaciones, valoraciones y expectativas sobre la formación docente, no existiendo disensos. Esto constituye un hallazgo relevante, por cuanto los discursos analizados pertenecen a estudiantes, adultos jóvenes y adultos mayores de ambos sexos, tanto mapuche como aymara, con muy distintos niveles de educación formal. Entre ellos existen pequeños agricultores con escasa educación formal, educadores tradicionales con educación básica, media y universitaria incompleta, profesores titulados en universidades tradicionales, uno de ellos con grado académico de Magister (mapuche) y otro con grado académico de Doctor (aymara). Entre los familiares y apoderados hay desde quienes asistieron muy poco a la escuela, hasta profesionales de áreas distintas a la educación. Esto demostraría que las concepciones, valoraciones y expectativas sobre la formación de quienes educarán a sus hijos e hijas están ancladas a la 
visión de mundo propia de sus culturas, con independencia del nivel de estudios formales que posean. Dicho de otro modo, a pesar de que la educación formal propicia otra visión de mundo, las y los colaboradores en estos estudios conservan sus conceptualizaciones y valoraciones tradicionales acerca de lo que consideran más importante que sus hijos aprendan de sus profesores, donde los contenidos del currículo son menos relevantes que el sentirse reconocidos en la legitimidad de su pertenencia a una cultura distinta, a partir de los modos de interacción que el profesor o profesora debiese propiciar en el aula.

\section{CONCLUSIONES}

Relevar la educación intercultural como elemento transversal en la escuela en general y en la formación docente en particular, no significa dirigir esa educación a las personas que pertenecen a culturas minoritarias, sino proporcionar una oportunidad real para mejorar la calidad de la educación de todos y todas, a partir de comprender el significado que tiene la interculturalidad para quienes pertenecen a culturas minoritarias. Como bien se señala en algunas expresiones de los participantes en el estudio, somos nosotros, miembros de la cultura occidental dominante y de tradición homogeneizadora, quienes nos enriqueceríamos al contar con espacios y experiencias para vivir y convivir con otras culturas, desde una relación de mutua legitimación.

La incorporación de la interculturalidad en la formación de profesores no puede realizarse simplemente en base a la creación de una cátedra de interculturalidad como se ha hecho con otras temáticas de relevancia; es necesario hacerse cargo de lo fundamental y transversal que resulta ser la interculturalidad en la formación de profesionales de la educación en el contexto actual de nuestro país e incorporarla como eje transversal en el currículo de formación. Lograr lo anterior significaría un comienzo importante porque, si bien la formación docente sería la que podría tener mayor incidencia en un cambio en el modo en el cual lo intercultural y particularmente lo indígena son percibidos por la sociedad chilena, es necesario realizar esfuerzos a nivel más general. El diálogo entre culturas en igualdad de condiciones, debiese ser nuestra meta para la relación día a día, para la interacción cotidiana entre ciudadanos. Como expresó una profesora mapuche,

entonces, o hacemos cultura occidental o hacemos cultura mapuche o logramos equilibrar estas dos culturas pero en forma de respeto, pero darle el mismo realce, el mismo realce [,,,] porque si queremos sobrepasar una, siempre la que está más arriba es la que va a ganar ¿y el resto?

Convivir interculturalmente en una relación que mire al otro en su legitimidad, implica generar espacios y experiencias para esa convivencia. Esto requiere, además de la disposición emocional favorable para realizarlo, la comprensión de que existen modos distintos pero igualmente legítimos de construcción y validación de conocimientos, los que se configuran a partir de lo que las personas de una determinada cultura viven y del sentido que dan a sus experiencias (Long 1992). La incorporación de competencias transversales para trabajar pedagógicamente la diversidad cultural en el aula, haría factible la generación de propuestas innovadoras que rompan esquemas técnicos e instrumentales que impiden mirar la riqueza de la diversidad escondida en el afán por sostener una única verdad sobre la 
realidad y trabajar sobre la idea, de suyo insostenible, de que todas y todos los estudiantes han tenido procesos de aprendizaje similares y comportan por tanto idénticos significados y sentidos para conceptos, objetos y relaciones.

Esperamos que los resultados preliminares del presente estudio contribuyan a la comprensión de lo que implica la interculturalidad para quienes no pertenecen a la cultura dominante o mayor, para avanzar en la inclusión de una concepción contextualizada y pertinente de la interculturalidad en la formación docente inicial y continua.

\section{REFERENCIAS BIBLIOGRÁFICAS}

Alarcón, Patricio. (2005). Hacia una pedagogía de la coexistencia. Revista de Pedagogía Critica Paulo Freire (3), 63-84. DOI: http://dx.doi.org/10.25074/07195532.3.511

Alegría, M. y Cisternas, T. (2003). Significados y Saberes que Orientan las Acciones Educativas de Estudiantes de Pedagogía de la Universidad Metropolitana de Ciencias de la Educación. Ponencia. Actas Congreso FID 2003.Santiago:UMCE.

Alvarez, C. (2012) La relación teoría-práctica en los procesos de enseñanza-aprendizaje. Educatio Siglo XXI, 30, (2), 383-402.

Aymerich, J.; Canales M y Vivanco, M. (2003) Encuesta Tolerancia y No Discriminación.. Santiago: FACSO/Universidad de Chile.

Carihuentro, S. (2007). Saberes mapuche que debiera incorporar la educación formal en contexto interétnico e intercultural según sabios mapuche. Tesis de Magíster en Educación con Mención en Currículum y Comunidad Educativa. Santiago: Universidad de Chile.

Casassus, J. (2007). La educación del ser emocional. Santiago: Cuarto propio.

Comisión Económica para América Latina y el Caribe (2014). Los pueblos indígenas en América Latina; Avances en el último decenio y retos pendientes para la garantía de sus derechos. Naciones Unidas:CEPAL.

CEPAL (2016) Panorama Social y Económico Suramericano. Naciones Unidas: CEPAL.

Claude, M. (2011). La educación pública es posible. Santiago: Universidad de Chile. Recuperado el 30 de abril de 2012 desde www.archivochile.com/Chile_actual/01_mse/5/MSE5_0016.pdf

Crickmay, Lindsey (2002). Transmission of knowledge through textiles: Weaving and learning how to live. In Stobart, Henry, Howard, Rosaleen (Eds.) Knowledge and Learning in The Andes: Ethnographic Perspectives (pp.38-50). Published Liverpool: Liverpool University Press.

Comisión Europea. (2002). Informe Comisión Europea. Educación y formación en Europa: sistemas diversos, objetivos compartidos para 2010. Luxemburgo:Comisión Europea. Recuperado el 27 de abril de 2003 desde http://www.peretarres.org/eutses/recursos/educacion.pdf

Díaz, T. y Druker, S. (2007). La democratización del espacio escolar: Una construcción en y para la diversidad. Revista Estudios Pedagógicos, XXXIII, (1), 63-77. DOI: https://dx.doi.org/10.4067/ S0718-07052007000100004

Druker, S. (2010). Educación, cultura e interculturalidad en el trabajo de Juan Ansión. AKADÉMEIA, $1(1), 41-54$.

Encuesta de Caracterización Socioeconómica. (2015). Casen 2013 Pueblos Indígenas. Santiago: Ministerio de Desarrollo Social.

Etxeberría, X. (2003). La ciudadanía de la interculturalidad. En N. Vigil y R. Zariquiey (Eds.), Ciudadanías inconclusas, (pp. 91-110). Lima: GTZ Cooperación Técnica Alemana-Pontificia Universidad Católica del Perú.

Flórez, O., R. (1994). Hacia una pedagogía del conocimiento. Caracas: Mc Graw Hill.

Gibbs, G. (2012). El análisis de datos cualitativos en investigación cualitativa, Madrid: Morata.

Goetz, J. y LeCompte, M. (1988). Etnografía y diseño cualitativo en investigación educativa. 
Estudios Pedagógicos XLIV, N 1: 225-239, 2018

INTERCULTURALIDAD EN LA FORMACIÓN DOCENTE: UN APORTE DESDE LAS VOCES DE PERSONAS DE LOS PUEBLOS ORIGINARIOS

Madrid: Morata.

Habermas, J. (1994). Teoría de la acción comunicativa: complementos y estudios previos. Madrid: Cátedra.

Ibáñez, N. (2002). Las emociones en el aula. Revista Estudios Pedagógicos, (28), 31-45. DOI: https:// dx.doi.org/10.4067/S0718-07052002000100002

Ibáñez, N. (2014). Saberes profesionales para la transformación del modelo educativo en Chile. Revista Estudios Pedagógicos, 40 (Especial), 145-160. DOI: https://dx.doi.org/10.4067/S071807052014000200009

Ibáñez, N., Barrientos, F., Delgado, T., Figueroa, A., \& Geisse, G. (2004). Las emociones en el aula y la calidad de la educación. Pensamiento Educativo Revista de Investigación Educacional Latinoamericana , 35 (2), 292-310.

Ibáñez, N.; Díaz, T.; Druker, S.; Rodríguez, M:S: (2012). La comprensión de la diversidad en interculturalidad y educación. Convergencia Revista de Ciencias Sociales, 19 (59), 215-240.

Instituto Nacional de Derechos Humanos. (2011). Primera Encuesta Nacional de Derechos Humanos. Santiago: INDH.

Jáuregui, M., Carrasco, L. y Montes, I. (2003). Evaluando, evaluando: ¿Qué piensa y qué hace el docente en el aula? Informe Final de Investigación. Arequipa: Universidad Católica Santa María. Recuperado el 12 de abril de 2006 desde http://cies.org.pe/files/active/0/R0204.pdf10.

Kvale, S. (2011). Las entrevistas de investigación cualitativa. Madrid:Morata.

Leiva, Juan J. (2009). "El profesorado ante la concepción de los conflictos en contextos de educación intercultural". Aula Abierta, 2 (37), 93-110.

Long, N. (1992). Battlefields of knowledge. London and New York: Norman Long and Ann Long.

Magendzo, Abraham. (2000). La diversidad y la no discriminación: Un desafío para una educación moderna. Pensamiento Educativo, (26), 173-200.

Maturana, H. (1990). Emociones y lenguaje en educación y política. Santiago: Dolmen. . (1992). El sentido de lo humano. Santiago: Hachette.

Mercado, Ruth (2002). Los saberes docentes como construcción social. México: Fondo de Cultura Económica.

MINEDUC. (2005). Informe de la Comisión sobre Formación Docente Inicial. Santiago:Mineduc.

MINEDUC. (2014). Resultados SIMCE 2013.Santiago: Mineduc.

MINEDUC. (2016). Recomendaciones para una Política Nacional de Desarrollo Curricular. Santiago: Mineduc/ Unidad de Curriculum y Evaluación.

Mizala, A. (2007). La economía política de la reforma educacional en Chile. Serie Estudios SocioEconómicos, (36), 1-45. Santiago: CIEPLAN. Recuperado el 27 de diciembre de 2007 desde www.cieplan.org/media/.../153/Capitulo_1.pdf

Moon, B. (2002). La Formación Docente en Inglaterra: perspectiva nacional e internacional. En Formación Docente: un aporte a la discusión, (pp.148-167). Santiago: UNESCO.

Mora, M. (2012). Educación Superior, Interculturalidad y Legislación en Chile: Relaciones Pospuestas. En D. Mato (Coord.), Educación Superior y Pueblos Indígenas y Afrodescendientes en América Latina. Normas, Politicas y Prácticas (pp.211-244). Caracas: IESALC-UNESCO,

Naciones Unidas (2009). Informe de Chile ante el Comité para la Eliminación de la Discriminación Racial de la Convención Internacional sobre la Eliminación de todas las formas de Discriminación Racial. Recuperado el 14 de septiembre de 2009 de http://acnudh.org/wpcontent/uploads/2009/09/CERD-CHILE-2009.pdf

Naciones Unidas (2013). Observaciones finales respecto de los informes periódicos $19^{\circ}$ a $21^{\circ}$ de Chile al CERD. Recuperado el 10 de octubre de 2013 de: http://docstore.ohchr.org/

Ñanculef, J. (2016). Tayiñ mapuche kimün Epistemología mapuche - Sabiduría y conocimientos. Santiago: Universidad de Chile.

Observatorio Ciudadano (2013). Informe de la sociedad civil y pueblos originarios de Chile al CERD. Recuperado el 5 de noviembre de 2013 de http://tbinternet.ohchr.org/Treaties/CERD/ 
Shared\%20Documents/CHL/INT_CERD_NGO_CHL_14779_S.pdf

OCDE. (2004). Informe de la Educación en Chile. Santiago: MINEDUC/OCDE.

Organización para la Cooperación y el Desarrollo Económico. (2010). Educating Teachers for Diversity: Meeting the Challenge. París:OCDE Publishing.

Oficina Regional de la Internacional de la Educación para América Latina. (2003). La privatización de la educación en Chile. Costa Rica:ORIEAL.

Paredes, R. y Pinto, J.I. (2009). ¿El fin de la educación pública en Chile?. Estudios de Economía, 36, (1), 47-66.DOI: https://dx.doi.org/10.4067/S0718-52862009000100003

Programa de las Naciones Unidas para el Desarrollo (2013). Primera Encuesta de Relaciones Interculturales 2012. Santiago: PNUD.

Pinto, R. (2008). El currículo necesario para una época compleja. Revista F@ ro. Año 4 (8), 1-8. Recuperado el 15 de junio de 2016 de http://web.upla.cl/revistafaro/05_resenas/08_index.html

Preiss, D; Calcagni, E.; Espinoza, A.; Gómez, D.; Grau, V.; Guzmán, V.; Müller, M.; Ramírez, F y Volante, P. (2014). Buenas prácticas pedagógicas observadas en el aula de segundo ciclo básico en Chile. Psykhe, vol.23 (2), 1-13. Recuperado el 20 de marzo de 2016 desde http://www. scielo.cl/scielo.php?script=sci_arttext\&pid=so718-22282014000200002 DOI: https://dx.doi. org/10.7764/psykhe.23.2.734

Quilaqueo, D. (2007). Diversidad étnico - cultural y educación intercultural cimentada en la interacción de saberes. Revista Latinoamericana de educación inclusiva, 1 (1), 37-46.

Quintriqueo, S., Quilaqueo, D., Lepe-Carrión, P., Riquelme, E., Gutiérrez, M. y Peña-Cortes, F. (2014). Formación del profesorado en educación intercultural en América Latina. El caso de Chile. Revista Electrónica Interuniversitaria de Formación del Profesorado, 17 (2), 201-217. Recuperado el 3 de julio de 2015 desde https://dialnet.unirioja.es/servlet/revista?codigo=2484 DOI: http://dx.doi.org/10.6018/reifop.17.2.198021

Redón, S., Toledo, L. y Rubio, G. (2009). La vivencia de "lo común" en la escuela: Prácticas y configuraciones de representación simbólica de cohesión social y construcción de comunidad en niños y niñas de cuatro a diez años de edad. Informe de investigación Proyecto FONIDE. Recuperado el 10 de marzo de 2011 desde http://www.fonide.cl/DecPublico/documentos

Romero, J. Luis, A.; García, F y Rozada J. (2007). La formación del profesorado y la construcción social de la docencia. En J. Romero y A. Luis (Eds.) A la luz de una "profesionalidad democrática”, (pp.8-77). Cantabria: Consejería de Educación de Cantabria.

Tardif, M (2004). Los saberes del docente y su desarrollo profesional. Madrid: Narcea

Tardif, M. et Lessard, C. (2004). La profession d'enseignant aujourd'hui. Bruxelles: De Boeck Université.

Turra, O. (2012). Currículo y construcción de identidad en contextos indígenas chilenos Educación y Educadores, vol. 15 (1), 81-95.

Shulman, L. (2005) El saber y entender de la profesión docente. Estudios Públicos, (99), 195-224.

UNESCO. (2000). Primer Estudio Internacional Comparativo sobre Lenguaje, Matemática y factores asociados para alumnos del tercer y cuarto grado de la educación básica. Santiago: UNESCO/OREALC.

UNESCO. (2008). Primer Reporte. Segundo Estudio Regional Comparativo y Explicativo. Los aprendizajes de los estudiantes de América Latina y el Caribe. Santiago: UNESCO /OREALC.

Valenzuela, I. (2006). Hacia la construcción del saber especializado del profesor: el saber pedagógico. Tesis para optar al grado de Magíster en Educación. Valparaíso:Universidad Católica de Valparaíso.

Webb, A, Canales, A. y Becerra, R. (2017). Las desigualdades invisibilizadas: población indígena y segregación escolar. En Propuestas para Chile. Concurso Políticas 2016, (pp.279-305). Santiago: PUC. 
